\title{
On Solitons, Non-Linear Sigma-Models, and Two-Dimensional Gravity
}

\section{Floyd L. Williams*}

Department of Mathematics and Statistics, Univ. Massachussets, Massachussets, USA

E-mail: williamsemath.umass.edu

Some interesting inter-connections between solitons, non-linear sigma-models, and gravity (in two and four dimensions) are discussed. Certain sigma-models and non-constant scalar curvature metrics are constructed from generalized solitons. Speculation is presented whether such metrics can be transformed ( by a suitable change of coordinates) to black hole metrics.

Fourth International Winter Conference on Mathematical Methods in Physics 09 - 13 August 2004

Centro Brasileiro de Pesquisas Fisicas (CBPF/MCT), Rio de Janeiro, Brazil

\footnotetext{
* Speaker.
} 


\section{Introduction}

Various connections between non-linear sigma-models and gravity have been the subject of discussion for some forty years or so; compare $[1,2,3,8,10,11,12,13]$. Recent discussions have involved links between solitons and gravity $[5,6,7,16,17]$. We extend these discussions here, where a link between solitons, sigma-models, and two-dimensional Jackiw-Teitelboim gravity is presented. Also presented is a construction of sigma-models (specifically maps of the plane to the 2-sphere), given solitons of a generalized type, and we construct corresponding metrics that we propose should be of interest for more general theories of two-dimensional dilaton gravity. Some background material on constant curvature metrics and sine-Gordon solitons is included before generalized sine-Gordon equations are considered.

The author expresses his sincere gratitude to members of the Organizing Committee for the opportunity to participate in this outstanding Winter School and to the dear people of CBPF for their kind assistance in many matters.

\section{Constant curvature metrics, sine-Gordon solitons, and two-dimensional gravity}

The connection between constant curvature metrics and solutions of sine-Gordon equations is reviewed here, with the introduction of some notation. For a pseudo Riemannian manifold $(M, g)$ with local expression

$$
d s^{2}=\sum_{i, j=1}^{m=\operatorname{dim} M} g_{i j} d x_{i} d x_{j}
$$

of the metric $g$, we shall observe the following sign convention for the curvature tensor $R_{i j k}^{l}$ and the scalar curvature $R=R(g)$ of $g$, where $g^{-1}=\left[g^{i j}\right]$ :

$$
\begin{aligned}
R_{i j k}^{l} & =\frac{\partial \Gamma_{j k}^{l}}{\partial x_{i}}-\frac{\partial \Gamma_{i k}^{l}}{\partial x_{j}}+\sum_{p=1}^{m}\left[\Gamma_{i p}^{l} \Gamma_{j k}^{p}-\Gamma_{j p}^{l} \Gamma_{i k}^{p}\right] \\
R & =\sum_{i, j=1}^{m} g^{i j} R_{i j} .
\end{aligned}
$$

Here

$$
R_{i j}=\sum_{l=1}^{m} R_{i l j}^{l}, \Gamma_{i j}^{k}=\frac{1}{2} \sum_{l=1}^{m} g^{l k}\left[\frac{\partial g_{i l}}{\partial x_{j}}+\frac{\partial g_{j l}}{\partial x_{i}}-\frac{\partial g_{i j}}{\partial x_{l}}\right]
$$

are the Ricci tensor and Christoffel symbols, respectively, of $g$. The Gaussian curvature $K$ of $g$ is $K=-R / 2$. We will have an interest in the particular case when $M$ is two-dimensional: $m=2$. In this case one always has the formula $R_{i j}=(R / 2) g_{i j}$. That is, the Einstein vacuum equations $R_{i j}-\frac{R}{2} g_{i j}+\Lambda g_{i j}=0$ automatically hold for a vanishing cosmological constant $\Lambda$. These equations consequently are of less interest and one considers instead the non-trivial Einstein equation (in two dimensions)

$$
R(g)=A(\text { a constant })
$$


due to Jackiw-Teitelboim (J-T) $[9,15]$. Equation (2.4) is derived from the J-T action integral

$$
I_{\mathrm{J}-\mathrm{T}}(\tau, g)=\frac{1}{2 G} \int_{M} \sqrt{|\operatorname{det} g|} d x_{1} d x_{2}(A-R(g)) \tau
$$

by variation of a scalar field $\tau\left(x_{1}, x_{2}\right)$ (called a dilation). The example

$$
\tau(x, t) \stackrel{\text { def }}{=} \sqrt{1+v^{2}} \operatorname{sech}\left[\frac{m(x-v t)}{\sqrt{1+v^{2}}}\right]
$$

(with $\left.\left(x_{1}, x_{2}\right)=(x, \tau)\right)$ appears in section 3 of [6].

Given $A$, solutions $g$ of equation (2.4) can be obtained on the basis of a well-known observation, where we denote the coordinates $\left(x_{1}, x_{2}\right)$ by $(x, y)$ : For a function $f(x, y)$, the metric $g$ defined by

$$
d s^{2}=\cos ^{2}(f(x, y)) d x^{2}+\sin ^{2}(f(x, y)) d y^{2}
$$

(with $g_{12}=g_{21}=0$ ) has scalar curvature

$$
R(g)=4\left(f_{x x}-f_{y y}\right) / \sin 2 f .
$$

This follows by (2.3),(2.4), or more directly by the formula

$$
\begin{aligned}
R= & \frac{2}{g_{11} g_{22}} R_{1212} \stackrel{\text { i.e. }}{=} \frac{2}{g_{11} g_{22}}\left[\frac{1}{2} \frac{\partial^{2} g_{11}}{\partial y^{2}}+\frac{1}{2} \frac{\partial^{2} g_{22}}{\partial x^{2}}-\frac{1}{4 g_{11}}\left(\frac{\partial g_{11}}{\partial y}\right)^{2}\right. \\
& \left.-\frac{1}{4 g_{22}}\left(\frac{\partial g_{22}}{\partial x}\right)^{2}-\frac{1}{4 g_{11}} \frac{\partial g_{11}}{\partial x} \frac{\partial g_{22}}{\partial x}-\frac{1}{4 g_{22}} \frac{\partial g_{11}}{\partial y} \frac{\partial g_{22}}{\partial y}\right]
\end{aligned}
$$

of Gauss (for any two-dimensional metric with $g_{12}=g_{21}=0$ ) [14]. Equation (2.8) means that $g$ in (2.7) is a solution of the Einstein equation (2.4) $\Longleftrightarrow f(x, y)$ is a solution of the sine-Gordon equation

$$
f_{x x}-f_{y y}=\frac{A}{4} \sin 2 f .
$$

Examples of solutions $f(x, y)$ of equation (2.10) (besides the trivial constant solutions $n \pi, n \in$ $\mathbb{Z}=$ the ring of integers) are the soliton ( solid wave) solutions:

1. $f(x, y)=2 \arctan [\exp (a(x-v y))]$ where $a=\left(1-v^{2}\right)^{-1 / 2}, A=2$-i.e. $K=-1$.

2. $f(x, y)=2 \arctan [\sinh (a v y) / v \cosh (a x)]$ for $a, A$ in example 1 . This is a soliton-antisoliton (or kink-antikink) soliton.

3. $f(x, y)=2 \arctan [a \sin (v y) / v \cosh (a x)]$ where $a=\left(1-v^{2}\right)^{1 / 2}, A=2$. This is a breather solution.

Of interest as well is a Euclidean version

$$
\Delta u \stackrel{\text { def }}{=} \frac{\partial^{2} u}{\partial x^{2}}+\frac{\partial^{2} u}{\partial t^{2}}=m^{2} \sin u(x, t)
$$


of the sine-Gordon equation. Here one considers the metric

$$
d s^{2}=\cos ^{2}\left(\frac{u}{2}(x, t)\right) d x^{2}-\sin ^{2}\left(\frac{u}{2}(x, t)\right) d t^{2}
$$

(with coordinates $\left(x_{1}, x_{2}\right)=(x, t)$ ), in contrast to the one in (2.7). Using the Gauss formula (2.9) one has, similarly, that $R=2 \Delta /(\sin u)$, and therefore this metric solves the Einstein equation (2.4) $R=2 m^{2} \Longleftrightarrow u$ satisfies (2.11). Here $m$ is any positive real number. For $v>0$ define

$$
a=a(v)=\left(1+v^{2}\right)^{\frac{1}{2}}, \rho(x, t)=\frac{m(x-v t)}{a(v)}, \beta(x, t)=\frac{m(v x+t)}{a(v)} .
$$

Then the dilation $\tau(x, t)$ in (2.6) is expressed as

$$
\tau(x, t)=a(v) \operatorname{sech} \rho(x, t)
$$

and

$$
u^{ \pm}(x, t) \stackrel{\text { def }}{=} 4 \arctan [\exp ( \pm \rho(x, t))]
$$

are the soliton solutions of (2.11) analogous to the solution $f(x, y)=2 \arctan [\exp a(x-v y)]$ of (2.10) in example 1. Moreover the functions $\Phi^{ \pm}: \mathbb{R}^{2} \rightarrow \mathbb{S}^{2}$ from the plane to the unit 2-sphere given by

$$
\Phi^{ \pm} \stackrel{\text { def }}{=}\left(\cos \beta \sin \alpha^{ \pm}, \sin \beta \sin \alpha^{ \pm}, \cos \alpha^{ \pm}\right)
$$

for $\alpha^{ \pm} \stackrel{\text { def }}{=} u^{ \pm} / 2$ are non linear $\sigma$-models -i.e. they are harmonic maps in the sense of J. Eells and J. Sampson[4]; see section 3. One has that

$$
\sin \alpha^{ \pm} \stackrel{\text { def }}{=} \sin \frac{u^{ \pm}}{2}=\operatorname{sech} \rho, \cos \alpha^{ \pm}=\mp \tanh \rho
$$

Consequently we can also write

$$
\Phi^{ \pm}=\frac{1}{a(v)}(\tau \cos \beta, \tau \sin \beta, \mp a(v) \tanh \rho) .
$$

Equations (2.16), (2.18) connect solitons $u^{ \pm}$, non-linear $\sigma$-models $\Phi^{ \pm}$, and two-dimensional gravity via the dilation $\tau$, where moreover the metric $g$ in (2.12) for $u=u^{ \pm}$satisfies the two-dimensional Einstein-Jackiw-Teitelboim field equation $R(g)=2 m^{2}$ (by the remark following (2.12)) and is known to transform to the black hole metric

$$
d s^{2}=-\left(m^{2} r^{2}-v^{2}\right) d T^{2}+\frac{d r^{2}}{\left(m^{2} r^{2}-v^{2}\right)}
$$

by a suitable change of variables $(x, t) \rightarrow(T, r)$. The explicit transformation $\Theta(x, t)=\left(\theta_{1}(x, t)\right.$, $\left.\theta_{2}(x, t)\right)=(T, r)$ of the metric (2.12) to the metric (2.19) is given, in fact, by

$$
\begin{aligned}
& \theta_{1}(x, t)=\frac{-1}{2 m v} \log \left[\frac{a(v) \tanh \rho(x, t)+1}{a(v) \tanh \rho(x, t)-1}\right]+\frac{x}{v}, \\
& \theta_{2}(x, t)=\tau(x, t) / m,
\end{aligned}
$$

which implements an observation of J. Gegenberg and G. Kunstatter [5,6], as discovered in [16,17]. 


\section{A generalization of the sigma-models $\Phi^{ \pm}$in equation (2.16)}

The main point of this section is to generalize the construction of the maps $\Phi^{ \pm}: \mathbb{R}^{2} \rightarrow \mathbb{S}^{2}$ in (2.16) in a way to produce new sigma-models. As the $\Phi^{ \pm}$were constructed via the solitons $u^{ \pm}$in (2.15), we shall seek first an appropriate replacement of these functions. We also consider the metric in (2.12) where $u$ is not necessarily a solution of the sine-Gordon equation, and the implication of such a metric for gravity.

For the sake of completeness, we define a harmonic map (or non-linear sigma-model) $\Phi$ : $(M, g) \rightarrow(N, h)$ of pseudo Riemannian manifolds. We proceed locally although a global, coordinate - independent definition is also available [4]. Let $\left(U, \phi=\left(x_{1}, \ldots, x_{m}\right)\right),\left(V, \psi=\left(y_{1}, \ldots, y_{n}\right)\right)$ be local coordinate systems on $M, N$ with $U \subset \Phi^{-1}(V)$ so that one can consider the $j^{\text {th }}$ coordinate functions $\Phi^{j} \stackrel{\text { def }}{=} y_{j} \circ \Phi \circ \phi^{-1}(1 \leq j \leq n)$ relative to these systems. We assume that $\Phi$ is a smooth map. Write $\partial_{j}=\frac{\partial}{\partial x_{j}}$ and let $\Delta_{g}$ denote the Laplace-Beltrami operator of $g$ :

$$
\Delta_{g} \stackrel{\text { def }}{=} \frac{1}{\sqrt{|\operatorname{detg}|}} \sum_{i, j=1}^{m} \partial_{i}\left[\sqrt{|\operatorname{det} g|} g^{i j} \partial_{j}\right]
$$

on $U$. If $\Gamma_{i j}^{k}$ are the Christoffel symbols of $h$ (see (2.3), with $g$ there replaced by $h$ ) then the nonlinear Laplacians $\tilde{\Delta}_{s}(1 \leq s \leq n)$ are defined to act on $\Phi$ by

$$
\left.\left(\tilde{\Delta}_{s} \Phi\right)(p) \stackrel{\text { def }}{=} \sum_{i, j=1}^{m}\left(g^{i j} \circ \phi^{-1}\right) \sum_{k, r=1}^{n} \partial_{i} \Phi^{k} \partial_{j} \Phi^{r}\right|_{\phi(p)} \Gamma_{k r}^{s}(\Phi(p))+\left.\Delta_{g} \Phi^{s}\right|_{\phi(p)}
$$

for $p \in U . \Phi$ is harmonic if it satisfies the system of equations

$$
\left(\tilde{\Delta}_{s} \Phi^{s}\right)=0,(1 \leq s \leq n=\operatorname{dim} N) .
$$

The field equation (3.3) can be derived by a variational principle where the energy integral of $\Phi$ is made stationary with respect to $\Phi$. For a Bosonic string, for example, this integral is the Polyakov integral and the equations (3.3) coincide with the equation of the motion of the string - say for $M=$ its two-dimensional world sheet and $N=\mathbb{R}^{26}, 26$ being the critical dimension. If $M \subset \mathbb{R}^{1}$ is some interval, then $\Phi$ is simply a smooth curve in $N$ and the equations (3.3) are the familiar conditions that $\Phi$ should be a geodesic. If $N$ is a flat space with vanishing Christoffel symbols $\Gamma_{i j}^{k}$ then the conditions (3.3) reduce to the standard conditions for harmonicity. In the case of $M=\mathbb{R}^{2}, N=\mathbb{S}^{2}$ with their standard Riemannian metrics, one has the following result. Given smooth functions $\alpha, \beta: \mathbb{R}^{2} \rightarrow \mathbb{R}$, the function $\Phi=\Phi_{\alpha, \beta}: \mathbb{R}^{2} \rightarrow \mathbb{S}^{2}$ defined by

$$
\Phi_{\alpha, \beta}=(\cos \beta \sin \alpha, \sin \beta \sin \alpha, \cos \alpha)
$$

is harmonic (-i.e. it satisfies conditions (3.3)) if $\alpha, \beta$ satisfy the conditions

$$
\begin{aligned}
\Delta \alpha \stackrel{\text { def }}{=} & \frac{\partial^{2} \alpha}{\partial x^{2}}+\frac{\partial^{2} \alpha}{\partial t^{2}}=\left[\left(\frac{\partial \beta}{\partial x}\right)^{2}+\left(\frac{\partial \beta}{\partial t}\right)^{2}\right] \sin \alpha \cos \alpha \\
& (\sin \alpha) \Delta \beta+2\left[\frac{\partial \alpha}{\partial x} \frac{\partial \beta}{\partial x}+\frac{\partial \alpha}{\partial t} \frac{\partial \beta}{\partial t}\right] \cos \alpha=0
\end{aligned}
$$


For example, $\alpha^{ \pm} \stackrel{\text { def }}{=} u^{ \pm} / 2$ and $\beta$ in (2.13) satisfy the system (3.5), since we have noted that $u^{ \pm}$ satisfy the Euclidean sine-Gordon equation (2.11). Hence one can conclude that $\Phi^{ \pm}=\Phi_{\alpha^{ \pm}, \beta}$ in (2.16) are harmonic maps, as asserted in section 2.

A fifth example, which nicely connects non-linear sigma-models and gravity ( this time fourdimensional gravity) is obtained by taking $M=\mathbb{R}^{2}, N=\mathbb{R} \times(\mathbb{R}-\{0\}) \times \mathbb{R} \times \mathbb{R}$,

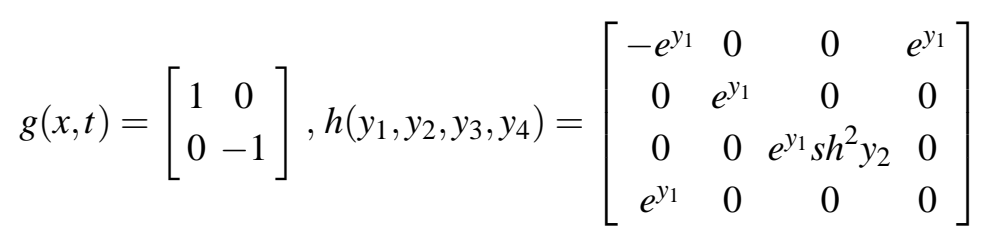

where $s h$ denotes the hyperbolic sine. $c t h$ similarly will denote the hyperbolic cotangent. The conditions (3.3) here ( where $s=4$ ) reduce to the following, where we write $\Phi=\left(\Phi^{1}, \Phi^{2}, \Phi^{3}, \Phi^{4}\right)$, $\Delta_{g}=\frac{\partial}{\partial x^{2}}-\frac{\partial}{\partial t^{2}}(=$ the Laplace- Beltrami operator of $g)$ :

$$
\begin{array}{r}
{\left[\Phi_{x}^{1}\right]^{2}-\left[\Phi_{t}^{1}\right]^{2}+\Delta_{g} \Phi^{1} \stackrel{(i)}{=} 0} \\
\Phi_{x}^{1} \Phi_{x}^{2}-\Phi_{t}^{1} \Phi_{t}^{2}-\frac{1}{2}\left[\left(\Phi_{x}^{3}\right)^{2}-\left(\Phi_{t}^{3}\right)^{2}\right] s h 2 \Phi^{2}+\Delta_{g} \Phi^{2} \stackrel{(i i)}{=} 0 \\
\Phi_{x}^{1} \Phi_{x}^{3}-\Phi_{t}^{1} \Phi_{t}^{3}+2\left[\Phi_{x}^{2} \Phi_{x}^{3}-\Phi_{t}^{2} \Phi_{t}^{3}\right] c t h \Phi^{2}+\Delta_{g} \Phi^{3} \stackrel{(i i i)}{=} 0 \\
\frac{1}{2}\left[\left(\Phi_{x}^{1}\right)^{2}-\left(\Phi_{t}^{1}\right)^{2}\right]-\frac{1}{2}\left[\left(\Phi_{x}^{2}\right)^{2}-\left(\Phi_{t}^{2}\right)^{2}\right]-\frac{1}{2}\left[\left(\Phi_{x}^{3}\right)^{2}-\left(\Phi_{t}^{3}\right)^{2}\right] s h^{2} \Phi^{2}+\Delta_{g} \Phi^{4} \stackrel{(i v)}{=} 0 .
\end{array}
$$

These equations follow by a direct computation of the Christoffel symbols of $h$ in (3.6); see Appendix 1. On the other hand, the conditions $(i),(i i),(i i i),(i v)$ (for $\Phi: M \rightarrow N$ to be a sigma-model) are exactly the Einstein gravitational equations for a 4-dimensional plane-symmetric space-time. Thus one has another beautiful connection between non-linear sigma-models and gravitation. This latter one is due to S.Chervon and A. Muslimov [1]; also see [2,3,13].

The key to generalizing the functions $u^{ \pm}$in (2.15), and hence the functions $\Phi^{ \pm}$, is the following very simple observation: The pair $(\rho, \beta)$ in (2.13) satisfies the Cauchy-Riemann (C-R) equations: $\rho_{x}=\frac{m}{a}=\beta_{t}, \rho_{t}=\frac{-m v}{a}=-\beta_{x}$. Thus $\rho$ and $\beta$ are harmonic conjugates. This observation motivates us now to choose $\rho$ to be any harmonic function on the plane $\mathbb{R}^{2}: \Delta \rho=\frac{\partial^{2} \rho}{\partial x^{2}}+\frac{\partial^{2} \rho}{\partial t^{2}}=0$. Since $\mathbb{R}^{2}$ is simply connected we now choose $\beta$ to be a harmonic conjugate of $\rho: \rho+\sqrt{-1} \beta$ is an analytic function. Motivated by (2.15) and the definition $\alpha^{ \pm}=u^{ \pm} / 2$, we define

$$
u(x, t) \stackrel{\text { def }}{=} 4 \arctan (\exp \rho), \alpha \stackrel{\text { def }}{=} \frac{u}{2} .
$$

One has (compare (2.17))

$$
\begin{gathered}
\sin \alpha=\operatorname{sech} \rho, \cos \alpha=-\tanh \rho, \\
\alpha_{x}=\frac{1}{2} u_{x}=\rho_{x} \operatorname{sech} \rho, \alpha_{t}=\frac{1}{2} u_{t}=\rho_{t} \operatorname{sech} \rho, \\
\Delta u=2(\Delta \rho) \operatorname{sech} \rho-2(\operatorname{sech} \rho \tanh \rho)\left(\rho_{x}^{2}+\rho_{t}^{2}\right)
\end{gathered}
$$


where $\Delta \rho=0$, by hypothesis. Therefore $\Delta \alpha=(\sin \alpha \cos \alpha)\left(\rho_{x}^{2}+\rho_{t}^{2}\right)=(\sin \alpha \cos \alpha)\left(\beta_{t}^{2}+\beta_{x}^{2}\right)$ (by the $\mathrm{C}-\mathrm{R}$ equations), which is the first equation in (3.5). The second equation there also holds since $\Delta \beta=0$ ( by definition of $\beta$ ), and since $\alpha_{x} \beta_{x}+\alpha_{t} \beta_{t} \stackrel{\text { def }}{=} \frac{1}{2}\left(\rho_{x} \beta_{x}+\rho_{t} \beta_{t}\right)=\frac{1}{2}\left[\rho_{x}\left(-\rho_{t}\right)+\rho_{t} \rho_{x}\right]$ (again by the C-R equations) $=0$. In summary we have therefore shown the following.

Theorem 1. Let $\rho(x, t)$ be any harmonic function on $\mathbb{R}^{2}: \Delta \rho=\rho_{x x}+\rho_{t t}=0$. Let $\beta(x, t)$ be a harmonic conjugate of $\rho(x, t)$. Define $u$ and $\alpha$ by (3.8). The $\alpha$ and $\beta$ are solutions of the system of equations in (3.5), and hence the function $\Phi_{\alpha \beta}: \mathbb{R}^{2} \rightarrow \mathbb{S}^{2}$ defined in (3.4) is non-linear sigmamodel - i.e. $\Phi_{\alpha \beta}$ satisfies the system of equations (3.3). Also $u$ satisfies the generalized type of sine-Gordon equation

$$
\Delta u=\left(\rho_{x}^{2}+\rho_{t}^{2}\right) \sin u
$$

(by (3.9)), which contrasts equation (2.11).

Consider the metric in (2.12) where we now take $u$ there to be the function in (3.8) for $\rho$ in Theorem 1. Denote this metric by $g_{\rho}$, which is a generalized type of soliton metric, given equation (3.10). By the remark following (2.12) it scalar curvature is given by $2 \Delta u /(\sin u)$, which by equation (3.10) equals $2\left(\rho_{x}^{2}+\rho_{t}^{2}\right): R\left(g_{\rho}\right)=2\left(\rho_{x}^{2}+\rho_{t}^{2}\right)$, which generally is non -constant -i.e. $g_{\rho}$ generally will not solve equation (2.4). One can determine all harmonic conjugate pairs $(\rho, \beta)$ for which $R\left(g_{\rho}\right)$ is a constant. Such pairs are given by $\rho(x, t) \stackrel{(i)}{=} a x-b t+c, \beta(x, t) \stackrel{(i i)}{=} b x+a t+d$ for suitable real numbers $a, b, c, d$ (which is consistent with the pair $(\rho, \beta)$ given in (2.31)). To see this, let $f=\rho+i \beta$ be the corresponding analytic function. Then $f^{\prime}=\rho_{x}+i \beta_{x}=\rho_{x}-i \rho_{t}$, by C-R, $\Rightarrow R\left(g_{\rho}\right)=2\left|f^{\prime}\right|^{2}$. In particular if $R\left(g_{\rho}\right)$ is a constant then $\left|f^{\prime}\right|$ is a constant, and since $f^{\prime}(z)$ is also analytic one may conclude that $f^{\prime}(z)$ is a constant: $\rho_{x}+i \beta_{x}=a+i b \Rightarrow \rho(x, t)=a x+c(t), \beta(x, t)=$ $b x+d(t)$, where by C-R, $a=\rho_{x}=\beta_{t}=d^{\prime}(t), c^{\prime}(t)=\rho_{t}=-\beta_{x}=-b \Rightarrow d(t)=a t+d, c(t)=$ $-b t+c$, which proves (i) and (ii).

Given the metric $g_{\rho}$, an obvious and very interesting question arises: Can one construct a transformation of variables $\Theta_{\rho}: \mathbb{R}^{2} \rightarrow \mathbb{R}^{2},(x, t) \rightarrow(T, r)$, under which $g_{\rho}$ goes (perhaps) to a black hole metric $G_{\rho}$ (as we did in (2.20) in the constant curvature set-up $R=2 m^{2}$ )? One would like $G_{\rho}$ to assume the form

$$
d s^{2}=A(r) d T^{2}-\frac{d r^{2}}{A(r)},
$$

for example; compare (2.19). The latter metric has scalar curvature $-A^{\prime \prime}(r)($ by(2.9)). In this more general setting we replace the J-T action integral given in (2.5) by

$$
I(g, \tau)=\frac{1}{2 G} \int_{M} \sqrt{|\operatorname{det} g|} d x_{1} d x_{2}[V \circ \tau-R(g) \tau]
$$

with equations of motion

$$
\begin{array}{ll}
R(g)=\frac{d V}{d x_{2}} \circ \tau & (\text { varying } \tau), \\
\Delta_{g} \tau+V \circ \tau=0 & (\text { varying } g),
\end{array}
$$


where $V$ is a function of $x_{2}$ only and $\Delta_{g}$ is given by (3.1); see [7]. Hopefully, future work will lead to a construction of $\Theta_{\rho}$. We note in fact that for the coordinates $\left(x_{1}, x_{2}\right)=(T, r)$, the metric $G_{\rho}$ in (3.11) and the dilation $\tau(T, r) \stackrel{\text { def }}{=} r$ do provide a solution of the field equations (3.13), for $A^{\prime}(r)=V(r)$. This follows from (3.1) which for $g=G_{\rho}$ gives

$$
\Delta_{G_{\rho}}=\frac{1}{A(r)} \frac{\partial^{2}}{\partial T^{2}}-A(r) \frac{\partial^{2}}{\partial r^{2}}-A^{\prime}(r) \frac{\partial}{\partial r} .
$$

Thus indeed $V^{\prime}(\tau(T, r))=A^{\prime \prime}(r)=-R\left(G_{\rho}\right)(T, r)$ (as noted in the line following (3.11)), which is the first equation in (3.13), and $\left(\Delta_{G_{\rho}} \tau\right)(T, r)+V(\tau(T, r))=-A^{\prime}(r)+A^{\prime}(r)=0$, which is the second equation in (3.13).

Note also that the function

$$
u_{2}(x, t)=4 \arctan \left[\frac{v}{\sqrt{1+v^{2}}}\left(\sinh \sqrt{1+v^{2}} m x\right) \sec v m t\right]
$$

is a solution of the Euclidean sine-Gordon equation $u_{x x}+u_{t t}=m^{2} \sin u$ in (2.11). This can be verified, for example, by a simple Maple program.

Given the solution (3.15), we can form the corresponding soliton metric $g_{u_{2}}$ in (2.12) which , in contrast to $g_{\rho}$, has constant curvature $R=2 m^{2}$ (again by the formula $R=2 \Delta /(\sin u)$ following (2.12)). Similar to the question posed for the metric $g_{\rho}$, it is meaningful to inquire whether one can construct a transformation $\Phi_{u_{2}}$ (as was done in (2.20) for the solitons in (2.15)) that realizes $g_{u_{2}}$ as a black hole metric. This is a question that my student, Miss S. Beheshti, is considering. The solution $u_{2}(x, t)$ is also called a kink-antikink solution. It describes a collision between a kink soliton and an antikink soliton.

\section{Appendix 1}

For the sake of completeness of the discussion in Sec. 3, we list the values of all the Christoffel symbols of the metric $h$ in (3.6). For $y=\left(y_{1}, y_{2}, y_{3}, y_{4}\right) \in \mathbb{R} \times(\mathbb{R}-\{0\}) \times \mathbb{R} \times \mathbb{R}, \Gamma_{11}^{1}(y)=1$, $\Gamma_{12}^{2}(y)=\frac{1}{2}, \Gamma_{33}^{2}(y)=\left(-\sinh y_{2}\right) \cdot \cosh y_{2}, \Gamma_{13}^{3}(y)=\frac{1}{2}, \Gamma_{23}^{3}(y)=\frac{\cosh y_{2}}{\sinh y_{2}}, \Gamma_{11}^{4}(y)=\frac{1}{2}, \Gamma_{22}^{4}(y)=-\frac{1}{2}$, $\Gamma_{33}^{4}(y)=\frac{-\left(\sinh y_{2}\right)^{2}}{2}$. All other symbols are zero ; of course one has the symmetry $\Gamma_{i j}^{k}=\Gamma_{j i}^{k}$.

\section{References}

[1] S. Chervon and A. Muslimov, Plane symmetric gravitational field as a four-component non-linear sigma-model, Phys. Lett. A142 (1989) 14.

[2] S. Chervon, D.Shabalkin, and V. Zhuravlev, Effective chiral model of a plane-symmetric gravitational field: properties and exact solutions, gr-qc/9810007.

[3] S. Chervon and D.Shabalkin, A plane-symmetric gravitational field with matter as a non-linear sigma model, gr-qc/0007001.

[4] J. Eells, Jr. and J. Sampson, Harmonic mappings of Riemannian manifolds, Amer. J. of Math. 86 (1964) 109. 
[5] J. Gegenberg and G. Kunstatter, Solitons and black holes, Phys. Lett. B413(1997) 274 [hep-th/9707181];

J. Gegenberg and G. Kunstatter, Sine-Gordon solitons and black holes, hep-th/9709183.

[6] J. Gegenberg and G. Kunstatter, From two-dimensional black holes to sine-Gordon solitions, In Solitons: Properties, Dynamics, Interactions, Application, Springer, Berlin 2000.

[7] J. Gegenberg, G. Kunstatter, and D. Louis-Martinez, Observables for two-dimensional black holes, Phys. Rev. D51 (1995) 1781.

[8] S. Iannus and M. Visinescu, Spontaneous compactification induced by non-linear scalar dynamics, gauge fields and submersions, Class. and Quantum Grav. 3 (1986) 889.

[9] R. Jackiw, Liouville FIeld theory: a two-dimensional model for gravity ?, In Quantum Theory of Gravity, Essays in honor of the 60th birthday of Bryce S. De Witt, Adam Hilger Ltd., Bristol 1984.

[10] R. Matzer and C. Misner, Gravitational field equations for sources with axial symmetry and angular momentum, Phys. Rev. 154 (1967) 1229.

[11] C. Misner, Harmonic maps as models for physical theories, Phys. Rev. D18 (1978) 4510.

[12] N. Sanchez, Harmonic maps in general relativity and quantum field theory, In Harmonic mappings, twisters, and sigma-models, edited by P.Gauduchon, World Scientific Pub., Singapore 1998.

[13] D. Shabalkin, On the method of effective non-linear sigma model in plane-and axially - symmetric vacuum space-times, gr-qc/9810053.

[14] D. Struik, Lectures on Classical Differential Geometry, Dover Pub., New York 1988.

[15] C. Teitelboim, The Hamiltonian structure of two-dimensional space-time and its relation with the conformal anomaly, In Quantum Theory of Gravity, Essays in honor of the 60th birthday of Bryce S. De Witt, Adam Hilger Ltd., Bristol 1984.

[16] F. Williams, Remarks on harmonic maps, solitons, and dilaton gravity, In Quantum Field Theory Under the Influence of External Conditions, Proceedings of the $6^{\text {th }}$ Workshop, Rinton Press, New York 2004.

[17] F. Williams, Further thoughts on first generation solitons and J-T gravity, to appear in Progress in Soliton Research, Nova Science Pub., New York. 